\title{
LOS ESTUDIOS ORTOGRÁFICOS DE NEBRIJA Y SU INFLUENCIA SOBRE EL ESTUDIO DE LOS IDIOMAS INDÍGENAS EN AMÉRICA
}

\author{
POR \\ Rosa Helena Chinchilla \\ University of Connecticut at Storrs
}

De vi ac potestate litteratum (Salamanca, 1503: 1981) y Reglas de la ortografia castellana (Alcalá de Henares, 1517: 1977) por Antonio de Nebrija forman la base teórica para el estudio de la ortografía en la época colonial. Los misioneros dedicados al aprendizaje de idiomas indígenas se inspiraron y guiaron por la obra de Nebrija Introductiones latinae que contenía distintas versiones de los tratados ortográficos. En el Reino de Guatemala los idiomas quiché, cakchiquel y tzutuhil fueron los más detalladamente estudiados en la época colonial. Arte de las tres lenguas kaqchiquel, k'iché y tz'utuhil (MS c. 1711: 1993) por Fr. Francisco Ximénez, O.P. y Arte de la lengua metropolytana del reino cakchiquel o Cuatimalico (Guatemala: Sebastián Arébalo, 1753) por Fray Ildefonso José Flores documentan las distintas controversias que surgieron alrededor de la ortografía de los idiomas desconocidos de América y que en el siglo XVIII se estudiaban científicamente. Estas gramáticas coloniales ejemplifican el problema de utilizar normas europeas para entender la multiplicidad lingüística de América.

La atención cuidadosa dedicada a aspectos de ortografía de las lenguas indígenas por autores coloniales de gramáticas se vincula la obra retórica de Quintiliano difundida por Nebrija en el siglo XVI. El estudio de los idiomas maya-quiché en el Reino de Guatemala se inició con los primeros misioneros franciscanos y dominicos que llegaron a catequizar a los pueblos de Guatemala después de 1524. La imprenta en México, y luego en Guatemala, trajo consigo la posibilidad de imprimir gramáticas para uso de misioneros no iniciados en estas lenguas. La invención de signos ortográficos, la creación de normas ortográficas y el método de difundir esta ortografía se volvieron cuestiones inherentes en esta época. El estudio cuidadoso de las teorías ortográficas ayuda a entender mejor las ideas científicas que circulaban en la época colonial y cómo influyeron en la comprensión de las nuevas lenguas encontradas por los mismoneros españoles.

El tratado más conocido de la ortografía del siglo XVI es Reglas de la orthografia castellana, el cual contiene esencialmente las mismas ideas que había enunciado Nebrija respecto al estudio comparativo de las lenguas clásicas. ${ }^{1}$ De vi ac potestate litteratum

\footnotetext{
${ }^{1}$ Entre los principales estudios de las Reglas de Nebrija se destacan el estudio en relación con la ortografia castellana de A. Alonso (1949) y la comparación con la Gramática Castellana de A. Sacks (1984). Se ha editado dos veces en el siglo XX por González Llubera (Oxford: Oxford University Press, 1926) y Antonio Quilis (1977).
} 
(Salamanca, 1503), formó parte de las ediciones de las Introductiones latinae que sí se reeditó a lo largo de los siglos XVI, XVII y XVIII. ${ }^{2}$ Su uso principal fue como texto de enseñanza de gramática latina en Salamanca y en las colonias americanas. Los privilegios reales de publicación en España y las colonias que contrataron los descendientes de Nebrija, cuyo oficio era el de impresores, se han documentado hasta el siglo XVIII (véase Matilla Tascón 1988).

La innovación principal acerca de la ortografia en los estudios de Nebrija era el principio de correspondencia exacta entre los sonidos y las letras. Nebrija en las Reglas de la ortografia castellana enumera siete definiciones en las cuales distingue qué es una letra y cómo se asignan las categorías de vocal, consonante, letra muda, semivocal, sílabas y diptongos (117-120). Añade a estas definiciones siete principios, de los cuales los principales para el estudio de las lenguas indígenas son los siguientes. El primer principio: "Que assí como los conceptos del entendimiento reponden a las cosas que entendemos, y assí como las bozes y palabras responden a los conceptos, assí las figuras de las letras han de responder a las bozes" (120). El tercer principio: "Que la diversidad de las letras no está en las figuras dellas, sino en la diversidad de la pronunciación" (121). El quarto principio: "Que anque las bozes humanas sean infinitas, porque los instrumentos y miembros donde se forman en infinitas maneras se pueden variar, cada lengua tiene ciertas y determinadas bozes, $y$, por consiguiente, ha de tener otras tantas figuras de letras para las representar" (122). El sexto principio sigue a Quintiliano y sustenta que como se toman las letras de diversas lenguas a veces sobran letras, a veces faltan, como cuando son consonantes, y otras veces se inventa el sonido que no existe en los alfabetos prestados (123). Y finalmente del séptimo principio la idea de que si las consonantes se doblaran será sólo en medio de las palabras (124). Con estos principios básicos los misioneros americanos "inventarán" o sea adaptarán el alfabeto romano y prestarán letras de otros alfabetos conocidos; y por consiguiente, muchas veces se encontrarán con letras demás, cuyo uso justificarán o deshecharán dependiendo de su filosofia ortográfica.

La fuente más antigua acerca de la pronunciación es el Cratilo de Platón. Cratilo arguye que la ortografia correcta no es una mera aproximación, porque sólo hay una manera de escribir un nombre correctamente. La importancia de la ortografia tiene mayor significado desde el punto de vista socrático. Amado Alonso arguye que Nebrija sostenía una actitud escolástica:

es la herencia de la filosofia escolástica que, buscando la armonización universal de todos los conocimientos, veía la instancia última del lenguaje no en la justificación de las formas por sí mismas ('En la lengua, todo es forma, nada es sustacia' Saussure), sino en la adecuación entre el lenguaje y la realidad; en la verdad, que tiene siempre su base en Dios. (6)

\footnotetext{
${ }^{2}$ De vi ac potestate litteratum contiene las ideas principales que Nebrija subdivide en De hebraicis litteris (1515) y De graecis litteris (1515). Se cree que el texto básico de esta ortografía comparativa de los idiomas clásicos ya la había presentado Nebrija en la Repetitio secundae (148?). El estudio de Alonso (1949) comenta estas obras también.
} 
Esa búsqueda de conformar la realidad desconocida con lo ya conocido va a mover a los gramáticos coloniales a buscar una correspondencia exacta entre los sonidos y las letras.

Instituto oratio (I iv-ix) de Quintiliano es la base de muchas ideas y actitudes que repite Nebrija. Quintiliano crea una guía para la pronunciación correcta de las letras del alfabeto. Él discute si el alfabeto es adecuado para representar los sonidos del idioma hablado, si contiene muchas o muy pocas palabras. Se explica allí cómo el gramático debe explicar las diferencias entre vocales y consonantes, las cuales se subdividen en mudas y semivocales. $Y$ finalmente el maestro debe familiarizar al estudiante con la ortografía antigua que pueda encontrar en textos antiguos. Se enfatiza que el gramático debe llamar la atención a los casos en que la ortografia no se conforma a la pronunciación. ${ }^{3}$ Las ideas de Quintiliano dominarán la idea de prescribir linguísticamente, la cual dominó la cultura europea por dos mil años (Harris y Taylor 72). Y son estas actitudes prescriptivas las que principalmente van a influir en la obra de José Ildefonso de Flores.

Nebrija, como los misioneros americanos, quería explicar la pronunciación, y por ende, la ortogría de los idiomas desconocidos. Nebrija se limita a discutir los idiomas de la antigüedad cristiana ya casi desconocidos en el siglo XV: el griego, el latín y el hebreo.

Si Nebrija no describe los sonidos modernos no es porque no sea capaz, sino porque no lo juzga necesario o pertinente: él describe los sonidos antiguos, para enseñarlos a los modernos; los sonidos castellanos todos los saben y habla de ellos "como de cosas conocidas"; el describe los sonidos clásicos, como materia de plena dignidad de estudio.

(Alonso 18)

Para Nebrija el estudio de la ortografia residía en la necesidad de enseñar la pronunciación correcta al estudioso de los idiomas de la antigüedad. ${ }^{4}$ El gramático americano encuentra una justificación completa de su estudio de la ortografía de las lenguas extranjeras porque esto no formaba parte de lo conocido. La inaccesibilidad del idioma indígena vuelve justificable su estudio. Los misioneros tenían solamente el paradigma de los idiomas clásicos para aproximarse a la cuestión ortográfica, y fueron estos principios de Quintiliano, difundidos por Nebrija, los que siguieron estos misioneros en su estudio de los idiomas americanos.

Paralelamente a las primeras fases de la conquista americana se realizó la ocupación política y el dominio cultural del Reino de Granada. A. Alonso afirma que Nebrija no conocía el árabe (11). Y aunque lo discute brevemente en su tratado de 1503 no es aquí donde se podría haber sugerido el uso del árabe para estas letras necesarias a los americanistas. La única gramática que fue hecha en la fase inicial de la conquista de Granada, comisionada por el Obispo de Talavera, fue la obra de Pedro de Alcalá Arte para ligeramente saber la lengua árabe (Granada: Juan de Varela, 1505). Esta obra parece ser

\footnotetext{
${ }^{3}$ Harris y Taylor hacen un resumen muy perceptivo de las ideas de Quintiliano (69-72).

${ }^{4}$ Percival (1986) y Rico (1983) han confirmado que el conocimiento de la tradición hebrea que poseía, la alcanzó a través de los estudios importantes que realizó en la casa de Juan de Zúniga antes de 1492 .
} 
un modelo que siguieron los misioneros españoles en América. ${ }^{5}$ Tenía esencialmente las mismas funciones de enseñanza y catequización que querían adoptar los misioneros americanos. Alcalá escribió una gramática rudimentaria, seguida por una descripción de las letras del alfabeto árabe, la cual a su vez era suplementada por un catequismo básico y finalmente termina con un extenso vocabulario. ${ }^{6}$ Pedro de Alcalá dirige su obra a otros misioneros del reino de Granada y posiblemente del Norte de Africa.

Pedro de Alcalá ilustra muy claramente las letras del alfabeto árabe, pero por la mayor parte utiliza letras romanas para que el lector pueda fácilmente adquirir las nociones básicas del árabe. Explica el uso de letras que no provienen del alfabeto romano en la forma siguiente:

De manera que para la comun algaravia no ay necessidad de las saber ni conocer todas mas solamente quatro conviene saber. ka. dil. ce. ây. Cuyos sones no temenmos en nro. abc latino, ni menos con letras latinas se pueden suplir buenamente por tanto ay necessidad de conocerlas y sus caracteres y su boz y fuerça para conveniblemente pronunciar las palabras aravigas. $\mathrm{Y}$ aun para que dondequiera que en algun vocablo fueren menester puestas sobre las letras castellanas se les de su natural son y ponerse an en el vocabulario, y en todo lo que aquí se escriviere. (ciiii verso)

Los misioneros coloniales adoptarán esta misma actitud de utilizar solamente letras "inventadas" para sonidos totalmente ajenos al castellano. La coincidencia de las siglas usadas para la hache aspirada, tresillo y cuatrillo es tan evidente que sin lugar a dudas es éste el modelo que siguieron los misioneros americanos.

El inventor de estas nuevas letras fue el padre Francisco de la Parra (m. 1560), quien llega a la Nueva España en 1542 (Vázqueż, Crónica, t. I, 595). Él propone el uso de dos siglas que casi seguramente vienen del árabe: el tresillo, el cuatrillo, el cuatrillo con coma, la hache aspirada, y la sigla usada para enunciar el sonido tz. ${ }^{7}$ Las descripciones por Pedro de Alcalá de cómo se forman los sonidos ajenos al español coinciden muy de cerca con las descripciones de gramáticos en el Reino de Guatemala. Contando ya con el alfabeto adecuado, los gramáticos crearon cada vez obras más complejas que buscaban superar las obras de la primera fase del conocimiento de las lenguas indígenas.

\footnotetext{
${ }^{5}$ Ésta fue una de las más importantes obras del impresor Juan de Varela, cuya carrera se inicia en Granada bajo el patrocinato de Hernando de Talavera (Gallego Morell 21-23). Después trasladó su imprenta a Sevilla donde seguramente habrá tratado de distribuir copias de sus obras, entre las cuales se destacaba esta gramática. Y es posiblemente en Sevilla donde Parra obtuvo una copia de esta gramática.

${ }^{6}$ Es importante notar que la obra de Ximénez escrita a lo largo de las primeras tres décadas del siglo XVIII, todavía se rige por este patrón de organización.

${ }_{7}^{7}$ Las correspondencias son c y qu de la época colonial igual a $/ \mathrm{k} /$, tresillo equivalente a $/ \mathrm{q} /$, cuatrillo equivalente //, cuatrillo con coma equivalente a // la cual no se distingue de la tz. en muchas descripciones modernas del quiché (McQouwn 252). El cuatrillo no es precisamente un cuatro romano. Aparece en documentos manuscritos y en obras impresas de la época como la letra vau del árabe, y el tresillo proviene de la letra aín. A continuación se pondrán los nombres en corchetes donde aparece la letra impresa en el original.
} 
Las dos obras culminantes del estudio de los idiomas maya-quiché en el Reino de Guatemala fueron escritas en el siglo XVIII. Arte de las tres lenguas cakchiquel, quiché y tzutuhil, escrita por Fr. Francisco Ximénez O.P., forma parte de una obra magna que incluye una crónica del Reino de Guatemala, y principalmente para el estudioso moderno, la transcripción en quiché del Popol Vuh y su traducción literal al castellano. Ximénez tenía un amplio conocimiento de fuentes lingüísticas e históricas de los siglos XVI y XVII. Como padre párroco en las primeras fases de su carrera tuvo un contacto directo con los idiomas que él estudió, dentro de una población que casi no había tenido contacto con el español. El Arte de Ximénez se dirigía principalmente a otros padres párrocos que le siguieron en el convento dominico, y hay una fuerte crítica a sus predecesores por no haber creado anteriormente un instrumento de enseñanza útil. La segunda obra Arte de la lengua metropolytana del reino de Guatemala escrita por el franciscano, Fr. Ildefonso José de Flores, tuvo mayores repercusiones por haber sido publicada y difundida por todo el reino. Flores fue catedrático de cakchiquel en la Universidad de San Carlos de Guatemala de 1762 a 1772, por consiguiente su estilo emula los tratados lingüísticos de su época. Hay en su obra una mayor conciencia de crear un producto crítico con vistas a la docencia universitaria. Había decaído mucho el aprecio para los idiomas indígenas en la segunda mitad del siglo XVIII y finalmente se terminó la cátedra en 1813 (Lanning 22).

Ildefonso José de Flores toma una actitud científica basada en el modelo de Nebrija. En el primer capítulo titulado "De las letras características de este Ydioma y su buena pronunciación" define el alfabeto que se usará en su gramática. Éste está constituido en su mayor parte por letras que existen en el alfabeto romano, enumerando luego las letras que se han adaptado al idioma cakchiquel:

lo primero que se ha de advertir son las Letras con que se expressan sus voces; tiene pues este Idioma su alphabeto inadequadamente distinto del Castellano, por que conviene con él en unas Letras, y en otras se distingue. Conviene en la A. B. C. E. H. I. K. L. M. N. O. P. Q. R. T. V. X. Y.Z. y se distingue, assi en carecer de las Letras D. F. G. J. S. LL. como en añadir otras proprias, que son K. [cuatrillo], [cuatrillo con coma]. [tresillo]. [hache aspirada]. [tz]. (2)

La actitud científica permite que Flores tome un distanciamiento de su tema. Ésta es la realidad de la escritura y es todo lo que le importa.

En contraste, Ximénez en el primer capítulo titulado "De la pronunciacion, ortografia, y acento de estas lenguas" define el alfabeto de estas lenguas tomando en cuenta la pronunciación del hablante del idioma, o sea enfatizando el hecho de que la pronunciación castellana no se conforma con la pronunciación de los sonidos que estamos representando imperfectamente con las letras romanas:

aunque parece que no son del todo equivalentes; como la experiencia muestra, pues hablandole a un indio, y pronunciandole el vocablo, como se escrive, suele no entenderlo; aunque esto procede principalmente de no darle nosotros aquel aire y modo con que ellos lo pronuncian; y tambien, de que como ellos entre si sincopan, y cortan todos los vocablos al oirlos que se los pronuncian enteros, suelen estrañarlos, y dudar de si es aquello o otra cosa lo que se les dice, y tambien procede de su summa pusilanimidad; pero nos valemos de ellos por ser los mesmos o quasi, salva de la que totalmente careçen, que son: D. F. G. J. S. LL. Ñ. que estos nunca los pronuncian. (4) 
Ximénez entonces enfatiza aquellos sonidos de los cuales carecen los idiomas indígenas y en el resto del capítulo busca clarificar cuál es el sonido que corresponde a las demás letras.

El cuarto y sexto principio de Nebrija que tratan de la correspondencia de la figura y la invención de nuevas letras se ilustra en las diversas letras ajenas al castellano. Flores atribuye la invención de las siglas extranjeras al castellano al Pe. Parra; éstas eran tan adecuadas desde el punto de vista de los religiosos castellanos que continuaron su uso hasta principios del siglo $\mathrm{XX}:^{8}$

Las letras propias, y Caracteristicas de este Idioma, que no se hallan en ninguno otro, son las que con especialissimo cuydado, y reflexion inventó nuestro ingeniosissimo $\mathrm{P}$. Fr. Francisco de la Parra, tan propias para este Idioma, que parecen naturalmente expresivas de sus diversos conceptos. Son pues cuatrillo, cuatrillo con como tresillo, tz y cuatrillo con hache. (7)

Flores comenta sobre las letras en términos muy elogiosos. En cambio Ximénez hace hincapié en que algunos de estos sonidos se han "inventado" para facilitar la escritura del misionero. Pero incluso ante estas letras hay un deseo de reducir su número con fines prácticos: "Algunos caracteres han inventado los antiguos particulares, y propios para poder expresar la pronunciación propria de estos naturales que son [tresillo], [cuatrillo], [cuatrillo con coma] y [tz]" (5). Las letras que introdujó Parra eran ya de uso común, y necesarias para expresar las diferencias entre el castellano y los idiomas indígenas.

La importancia de estas letras se ilustra principalmente en el cuidado con el cual se describe la formación del sonido de cada una para establecer las diferencias significantes. Ximénez describe el tresillo de la siguiente manera: "denota que su pronunciación, es gutural, y se pronuncia dando como una castañeta en el gutur" (5). En cambio el cuatrillo lo describe como: "una c. que denota ser fuerte; con que se distingue [cuatrillo]a[cuatrillo], la pulga o nigua, de [tresillo]a[tresillo], el fuego, y de cac. la guayaba" (5). Ximénez añade el uso del cuatrillo con hache: "que es fuerte su pronunciacion [...] pero su uso es como en nuestro castellano decimos: chá, ché, chí [...]" (5). Y finalmente distingue el cuatrillo con coma: "y se pone por [tz]. fuertemente herida, a diferencia de quando se escribe tz que es mas suave su pronunciación que es el quarto tropo, o character" (5).

Ximénez además agrega el uso de $\mathrm{c}$. ante hache y las cinco vocales:

ora sea en esta figura [tresillo] ora en esta [cuatrillo] ora en esta $c$. ante puesta a la $h$ siempre se pronuncia como en el castellano decimos chimenea y no como solemos decir chimera. y quando esta letra c. en qualquiera figura que la escrivan, se antepone a estas letra A. O. U. siempre se queda conforme ella es. pero quando se le siguen esta E. I. se convierte en q. (5)

Ximénez quiere prever cualquier posible confusión que pueda haber para el lector castellano en el uso de las cuatro siglas y su combinación con vocales y hache. Flores

\footnotetext{
${ }^{8}$ La última obra impresa que usó estas siglas fue fr. Carlos J. Rosales, Gramática del idioma cakchiquel (Guatemala: Tipografia San Antonio, 1919).
} 
mantiene el uso de la $\mathrm{K}$ y lo distinque de la $\mathrm{C}$, pero no quedan explicados suficientemente en sus ejemplos:

La K, aunque tambien la tiene el Alphabeto Castellano, pero la pronuncia este Idioma distinto modo de los otros Idiomas; porque el Latino, Castellano la pronuncian suavemente, y como C v.g. Kalendae, como Calendae; pero el cakchiquel la pronuncia un poco aspera, abriendo un tanto los labios, y la lengua que no toque al paladar, ni abaxo, sino en el medio, y como enguecando la voca al proferirla. (6)

Hace diferencia con el cuatrillo de la siguiente manera: "es mas viva que la de K, y algo mas fuerte que la $\mathrm{C}$, hiere con la punta de la lengua al paladar castañeteando un poco" (78). Y luego procede a distinguir el tresillo: "es gutural, de manera que se pornuncia con fuerza castañeteando en el gutur, y se distingue de la [cuatrillo] en que aunque en esta se castañatea es un poco, con suavidad, y azia fuera del gutur como en la punta de la lengua; mas en la [tresillo] es con fuerza, y en el gutur" (8). Y finalmente describe la letra del cuatrillo con coma: "es una $\mathrm{C}$, muy viva pronunciase haziendo fuerza con la punta de la lengua, que se arrima algo mas a los dientes de arriba, que a los de abaxo, y se detiene algo en la pronunciación de ella" (10). Tanto Flores como Ximénez insisten en mantener la diversidad ortográfica porque es la misma pronunciación quien dicta esa diferencia. Esencialmente siguen los preceptos de Nebrija al mantener distincciones que para un falso docto tal vez serían superfluas.

El problema de las letras dobladas, ilustrado en el séptimo principio en la ortografia de Nebrija, también es tratado por Flores y Ximénez de manera diversa. Ximénez suprime del todo las letras dobladas creyéndolas innecesarias:

$\mathrm{Y}$ aunque algunos han querido añadir las siguientes $\mathrm{K}$. [hache con coma]. $\mathrm{tt}$. pp. tengo los por cosa superflua; porque la $\mathrm{K}$. es lo mesmo que una: C. comun. la [hache con coma] es lo mesmo que una: h. comun. y lo mesmo las dos tt. y las dos pp., sino que la diferencia que parece que ay está en los vocablos o consonantes que anteceden o se siguen que ellos mesmos compelen a que se pronuncien como debe ser. (5)

Ximénez también elimina la $\mathrm{K}$ y la hache aspirada para simplificar y aclarar la confusión que parecía existir. Flores está de acuerdo con Ximénez en que la t doblada es inútil, pero documenta su uso anterior: "La tt. geminada que algunos usan como Nro. Doctissimo P. Maldonado, que en varias partes de sus eloquentes Obras la escribe, como en el Sermon de la Samaritana [...] pero en orden a la pronunciacion lo mismo suena la tt doblada, que la sencilla, y assi esta Letra me parece superflua" (11). Al documentar su uso anterior Flores da cierta validez al uso de letras dobladas, tal vez por la influencia que tienen obras como las de Nebrija donde se hace hincapié en letras dobladas como un fenómeno frecuente de la ortografía.

Al igual que en la obra de Nebrija, las semivocales requieren párrafos explicativos. Ximénez trata muy ligeramente el problema de las semi-vocales: "y estas tres letras $h$. $X$. z. las tienen como semi vocales en quanto a las formaciones pues el Verbo o nombre, que acaba en estas letras, para lo que toca a formacion, no se entiende acabar en consonante sino en vocal como tratando de las formaciones se vera" (4). Y así aclara Ximénez cómo 
se clasificarán ese grupo de palabras a continuación en su gramática. Flores escribe sobra la semi-vocal hache: "La H. en este Idioma se escrive de dos maneras, sin vir[g]ula, con ella, v.g. [hache con coma]. h la sin vir[g]ula es para inicial, o media de dicción: la vir[g]ulada es precissamente para final" (4). Simplifica al final la regla de la $\mathrm{H}$. al volverla equivalente a todos los sonidos aspirados. Flores sostiene que así como en castellano hay letras demás, lo mismo puede ocurrir en estos idiomas.

que aunque los Naturales de este idioma usan en la escriptura de la $\mathrm{H}$. al pronunciarla es con mucha differencia de la nuestra Castellana, por que en el Castellano no es letra en rigor, sino aspiracion que podemos hablar sin ella, y entenderse la locucion; como Hijo. Yjo. Hermano. Ermano. Honor. Onor. Y en algunas dicciones donde podía sonar la $\mathrm{H}$, usan de G, o J, como Geometria, Generacion, Gitano, Gigante, Jamon, Jasinto, Jeremias, Job, Jugar, Juntar, y otras veces de X, como Xabon etc.; pero en este Idioma fozosissimamente se ha de pronunciar hiriendola con fuerza con si fueran dos gg. o dos jj. y esto sea ante vocal, o despues de vocal, de manera que siempre se ha de articular aspirando. (5)

Flores hace una argumentación fuerte para la clara definición de una hache aspirada que se pronuncia, y por eso concluye que debe mantenerse la letra en uso. Ximénez en cambio concluye que se debe eliminar del todo esta letra innecesaria y resuelve el problema así:

Tocante a la letra $\mathrm{h}$. y esta es una aspiracion como en todas las lenguas y no se puede pronunciar ni como j. ni como g. sino como en el latin decimos Vah quis destruis templum Dei. noten esto los que se precian de tan criticos que descubren su ignorancia y tosquedad [...] y haziendo nota de otros dizen: omo por homo; y ambre por hambre, es nota de aspiracion, $y$ lo mesmo es en estas lenguas, y el poner algunos la $h$. en esta forma [hache con coma]. no tiene substancia porque lo mesmo es una que otra, y solo está la diferencia en las vocales o consonantes que siguen. (5-6)

Ximénez parece tener más libertad de tachar sonidos que en su experiencia están de más. En cambio Flores es más conservador con respecto de mantener la ortografía tal como ha estado en uso, y por consiguiente justifica la práctica de escribir letras aspiradas.

Los dos autores no distinguen entre la letra $\mathrm{v}$ y la $\mathrm{u}$, así manteniendo la diferencia entre la semivocal. Ximénez está determinado a que se simplifique y regularice el uso de esta semi-vocal: "V. en estas lenguas, nunca sirve como consonante, sino, siempre como vocal. en el latin decimos: vanitas haciendo la $\mathrm{V}$. consonante en estas lenguas siempre es vocal" (6). Flores toma una actitud más inclusive acerca de las distinciones entre la semivocal v. El hecho de que su tratado sea impreso es importante, porque allí tampoco se hará una distincción entre v. y u.: "Y finalmente se advierte que la V. en esta lengua siempre se hiere quando esta ante vocal, aunque se escriba como v. consonante, a distincion de la lengua Latina, y Castellana que quando es consonante la pronuncian como b" (12).

Al igual que Ximénez, Flores insiste en concluir que no todas las letras son intercambiables, ni es su pronunciación sin importancia porque es en la escritura donde se refleja la diferencia para que el extranjero pueda encontrar la pronunciación correcta: "Aquí se advierta el cuidado que se debe tener en oir, y pronunciar las dicciones que se dicen con estas letras, por que en ellas esta la diversidad de significados y conceptos que hazemos de 
las voces" (9). Flores tanto como Ximénez advierte que la mala pronunciación puede llevar a errores mucho más graves si proviene del padre que enuncia la doctrina cristiana: "Y no aviendo cuidado en esto pueden engendrar en el auditorio ignorante, y nescio de los Indios algun concepto adverso a Nuestra Santa Feé, y Sagradas Doctrinas, y en los que fueren espertos alguna murmuracion, o burla" (10). Es la habilidad de poder decir las palabras correctamente, y por ende, de escribirlas correctamente, lo que mueve a Flores y Ximénez a incluir este capítulo en sus obras. Aunque es de notar que Ximénez inexplicablemente en un capítulo de su Arte decide uniformar todas las letras que empiezan con cuatrillo, tresillo o c. Es posible entonces pensar que todavía había cierta actitud laxa en el uso de estas letras, y es tal vez por eso que tanto Ximénez como Flores en sus párrafos introductorios quieren enfatizar esta cuestión.

Los fundamentos que expuso Nebrija en sus tratados ortográficos tuvieron muchas repercusiones, no sólo en el estudio del castellano, sino también en el estudio de los idiomas americanos en la época colonial. La necesidad de enseñar la doctrina religiosa y de predicar a los amerindios, posibilitó el estudio cuidadoso de estos idiomas. La cuestión ortográfica se presenta como uno de los problemas más agudos, porque aquí no se podía recurrir a estructuras ya hechas. Como se ha demostrado en el Reino de Guatemala los misioneros del siglo XVI tomaron de todos los manuales ortográficos a su alcance, y esta tradición estaba vigente todavía en el siglo XVIII. Ximénez, - dado su conocimiento práctico de la lengua, ofrece una guía amplia y directa, ejemplificada profusamente. Ximénez tiene una actitud escéptica en cuanto a sus co-gramáticos a quienes desprecia, por inexactos e inexpertos. Flores consciente de escribir para impresión crea un tratado que busca hacer un compendio de la erudición acerca del idioma cakchiquel, y por consiguiente valoriza en mayor grado a sus antecesores.

Las dos obras de Flores y Ximénez persisten en imponer en los idiomas americanos los principios de Quintiliano que dominaron los estudios ortográficos a lo largo de la historia occidental. Por su experiencia lingüística en algunos casos ellos indicaron mayor exactitud en sus descripciones, pero las convenciones de nombres y posibles usos en su mayor parte sólo se adaptaron a las circunstancias extraordinarias en sus tratados lingüísticos. Todavía en 1862 fueron estos dos pensadores a quienes Brasseur de Bourbourg recurrió extensamente para crear su gramática del quiché. La valorización y estudio de estas gramáticas se presenta como un ejemplo de cómo los misioneros americanos adaptaron ideas europeas para comprender y enseñar los idiomas de América.

ObRas CITADAS Y CONSULTADAS

Alcalá, Pedro de. Arte para ligeramente saber la lengua arábiga. Granada: Juan Varela de Salamanca, 1505.

Alonso, Amado. "Examen de las noticias de Nebrija sobre antigua pronunciación española". NRFH(1949)3: 1-82.

Bahner, Werner. La lingüistica del siglo de oro. Madrid: Editorial Ciencia Nueva, 1966. Bloch, Joshua. "Early Hebrew Printing in Spain and Portugal". Hebrew Printing and Bibliography. Charles Berlin, editor. New York: The New York Public Library and KTAV, 1976. 7-56. 
Brasseur de Bourbourg. Grammaire de la langue quichée. Paris: Auguste Durand, 1862. Cañes, fr. Francisco. Gramática arábigo-española. Madrid: Antonio Pérez de Soto, 1775.

Chinchilla Aguilar, Ernesto. La vida moderna en Centroamérica. Guatemala: Seminario de Integración Social, 1977.

"Fray Francisco Ximénez, el mayor de los lingüistas de Guatemala". Anales de la Sociedad de Geografia e Historia XXXIX (1966): 409-425.

Flores, fr. Ildefonso José. El arte de la lengua metropolytana del reino cakchiquel o Cuatimalico. Guatemala: Sebastián de Arébalo, 1753.

Fraenkel, Gerd. "The decline of Latin as a model for linguistic analysis". Proceedings of the 9th International Congress of Lingüistics Cambridge. Cambridge: Cambridge University Press, 1962. 730-7.

Gallego Morell, Antonio. Cinco impresores granadinos de los siglos XVI y XVII. Granada: Universidad de Granada, 1970.

Gil Fernández, Luis. Estudios de humanismo y tradición clásica. Madrid: Editorial de la Universidad Complutense, 1984.

"Nebrija y el menester del gramático". Academia literaria renacentista III: Nebrija. Salamanca: Universidad de Salamanca, 1983. 53-64.

Panorama social del humanismo español (1500-1800). Madrid, 1981.

Hazañas y La Rúa, Joaquín. La imprenta en Sevilla. Sevilla, 1945.

Harris, Roy y Talbot J. Taylor. Landmarks in Linguistic Thought: The Western Tradition from Socrates to Sausurre. London: Routledge, 1989.

Kukenheimer, Louis. 1951. Contributions à l'histoire de la grammaire grecque, latine, et hébraïque à l'époque de la renaissance. Leiden: Brill.

Lanning, John Tate. The Eighteenth Century Enlightenment in the University of San Carlos de Guatemala. Cornell: Cornell University Press, 1956.

Lemus y Rubio. "El maestro Elio Antonio de Lebrixa". Revue Hispanique 22 (1910): 459-508.

López Rueda, José. Helenistas españoles del siglo XVI. Madrid: Instituto Antonio Nebrija, 1973.

Matilla Tascón, Antonio. "Las impresiones de la 'Gramática' de Nebrija en los siglos XVII y XVIII”. Varia bibliographica: Homenaje a José Simón Díaz. Kassel: Reichenberger, 1988. 467-481.

McQuown, Norman. "Classical Quiché". Linguistics. Handbook of Middle American Indians. Austin, Texas: University of Texas Press, 1967. 251- 267.

Nebrija, Antonio de. De vi ac potestate litteratum. Antonio Quilis and Pilar Usabel, editores. Madrid: Sociedad General Española de Librería, 1988. (Salamanca, 1503). Diccionario latino-español. Salamanca, 1492. Introductiones latinae. Salamanca 1495. Lyon 1515. Granada, 1540. Reglas de ortografia en la lengua castellana. Antonio Quilis, editor. Bogotá: Instituto Caro y Cuervo, 1977. (Alcalá de Henares: Guillen de Brocar, 1517).

Odriozola, Antonio de. "La caracola del bibliófilo nebrisense". Revista de Bibliografia Nacional 7 (1946): 3-114.

Olender, Maurice. The Languages of Paradise. Cambridge: Harvard University Press, 1992. 
Padley, G.A. Grammatical Theory in Western Europe 1500-1700. The Latin Tradition. London: Cambridge University Press, 1976.

Padley, G.A. Grammatical Theory in Western Europe 1500-1700. The Vernacular Tradition. vols. I-II. London: Cambridge University Press, 1985, 1988.

Percival, W. Keith. "The reception of Hebrew in sixteenth century Europe: The Impact of the Cabbala". The History of Linguistics in Spain. Amsterdam: John Benjamins, 1986. 21-38.

"Renaissance Linguistics: the Old and the New". Studies in the History of Western Linguistics. Cambridge: Cambridge University Press, 1986. 56-68.

Quintillian. Institutes of Oratory. John Selby Watson, traductor. London: Bell, 1903.

Reyes, José Luis. "Conmemoración del tricentenario del nacimiento de fray Francisco Ximénez, O.P.”. Anales de la Sociedad de Geografia e Historia XXXIX (1966) 390391.

Ricard, Robert. La conquête espirituelle du Mexique. Paris: Institut d'Ethnologie, 1933. Rico, Francisco. "El nuevo mundo de Nebrija y Colón". Academia literaria renacentista III: Nebrija. Salamanca: Universidad de Salamanca, 1983. 157-185.

Nebrija frente a los bárbaros. Salamanca: Universidad de Salamanca, 1976. Robins, R.H. Ancient and Medieval Grammatical Theory. London: G. Bell \& Sons, 1951.

A Short History of Linguistics. London: Logman House, 1990.

Rodríguez Cruz, A. "La influencia de la Universidad de Salamanca en Hispanoamérica: aportación de los dominicos". Congreso internacional sobre los dominicos y el Nuevo Mundo. Madrid: Deimos, 1987. 641-674.

Rowe, John Howland. "Sixteenth and Seventeenth Century Grammars". Studies in the History of Linguistics, Traditions and Paradigms. Dell Hymes, editor. Bloomington: Indiana University Press, 1974. 361-379.

Sacks, Norman P. "Antonio de Nebrija: Founder of Spanish Linguistics". Hispanic Linguistics 1-2 (1984;1984) 1-33; 149-176.

Saénz de Santa María, Carmelo. "Dos grandes filólogos hispanoamericanos: Fray Francisco Ximénez, O.P. y Fray Ildefonso José Flores, O.F.M.”. Anales de la Sociedad de Geografia e Historia XVIII (1942) 122-132.

"Fray Francisco Ximénez, O.P.". Anales de la Academia de Geografia e Historia LVII (1983) 253-264.

"Introducción". Historia de la Provincia de San Vicente de Chiapa y Guatemala, Orden de Predicadores. Guatemala: Sociedad de Geografia e Historia, 1977.

Van Oss, Adriaan. Catholic Colonialism, a Parish History of Guatemala 1524-1821. Cambridge: Cambridge University Press, 1986.

Vázquez, Francisco. Crónica de la provincia del Santíssimo nombre de Jesús de Guatemala. 4 vols. Guatemala: Sociedad de Geografía e Historia, 1937-1944.

Ximénez, Francisco. Arte de las tres lenguas kaqchiquel, k'iché y tz'utuhil. Rosa Helena Chinchilla, editora. Guatemala: Academia de Geografía e Historia, 1993.

Escolios de la historia del origen de los Indios escoliados. Guatemala: Sociedad de Geografia e Historia, 1967.

Historia natural del Reino de Guatemala. Julio Roberto Herrera S., editor. Guatemala: Sociedad de Geografía e Historia, 1967. 
Historia de la Provincia de San Vicente de Chiapa y Guatemala. 3 vols. Guatemala: Sociedad de Geografia e Historia, 1929-1931. (Reprinted in its entirety by the Academia de Geografia e Historia de Guatemala 1971-1977).

Primera parte del Tesoro de las tres Lenguas Cakchiquel, Quiché y Zutuhil, en que las dichas Lenguas se traducen a la nuestra española. Carmelo Saénz de Santa María, editor. Guatemala: Academia de Geografía e Historia, 1985. 